\title{
Fast Segmentation of Range Images
}

\author{
Michal Haindl ${ }^{12}$ and Pavel Žid ${ }^{2}$ \\ 1 Center for Machine Perception, Czech Technical University, \\ 2 Institute of Information Theory and Automation of the Czech Academy of Sciences, \\ Praha, Czech Republic \\ E-mail: \{haindl,zid \}@utia.cas.cz
}

\begin{abstract}
A new type of range image segmentation method is introduced. The image segmentation is based on a recursive adaptive regression model prediction for detecting range image step discontinuities which are present at object face borders. Border pixels are detected in two perpendicular directions and detection results are combined together. Two predictors in each direction use identical contextual information from the pixel's neighbourhood and they mutually compete for the most optimal discontinuity detection. The method suggested can be successfully applied also to other image segmentation applications, e.g. panchromatic or multispectral image data, etc.
\end{abstract}

\section{Introduction}

Segmentation is a fundamental process affecting the overall performance of a machine vision system. Range image segmentation is a crucial part of autonomous navigation systems and as such it has been an active research area for past fifteen years. Segmentation of a range image should partion this image into meaningful patches representing single objects faces, but the task is complicated with outliers resulting from a sensing operation and corrupting boundary between distinct shapes. Optimal segmentation algorithm should be stable, accurate and numerically efficient.

There are many segmentation algorithms published in computer vision literature and a number of good survey articles [1],[10] is available. However their mutual comparison is very difficult because of lack of sound experimental evaluation results. A rare exception is results published in [7] together with experimental data available on their Internet server. These data and results are used also for our algorithm evaluation. The common approaches to solve the range image segmentation problem are region growing based algorithms [7],[2] where single regions are formed by iteratively growing from seed regions, splitand-merge, clustering (e.g. clustering in a fitted planes parameter space [4]), and edge based techniques [12]. Region growing and split-and-merge algorithms present the problem that they have to deal with different threshold values that are difficult to obtain (see [7]) and depend on an application. Clustering algorithms suffer usually less influence from this kind of problem, although other problems exist. Current segmentation algorithms most often miss small regions and perform poorly when the required region precision is high. Experiments in 
[7] indicate that missed and false detected regions occur more frequently than over or under segmentation.

The present paper is organised as follows. In Section 2, a proposed method general concept under a Bayesian framework is introduced. Section 3 completes the algorithm with a locally optimal model selection rule design. Section 4 deals with numerical realisation problems while Section 5 contains evaluation results obtained on range data test set [7].

\section{The Adaptive Regression Model}

The regression method uses high spatial correlation (and spectral in the case of multispectral segmentation applications) between neighbours of a predicted pixel. We assume the mono-spectral line to be modelled as:

$$
Y_{t}=P^{T} Z_{t}+E_{t}
$$

where $P^{T}=\left[a_{1}, \ldots, a_{\beta}\right]$ is the $1 \times \beta$ unknown parameter vector $\beta=\operatorname{card} I_{i}$. We denote the $\beta \times 1$ data vector

$$
Z_{t}=\left[Y_{t-i}: \forall i \in I_{t}\right]^{T}
$$

with a multi-index $\dot{t}=(m, n, d) ; Y_{t}$ is a predicted mono-spectral pixel value, $m$ is the row number, $n$ the column number, $d(d \geq 1)$ denotes the number of spectral bands, $E_{t}$ is the white noise component. $\bar{I}_{t}$ is some neighbour index shift set.

Note that although the model predicts a mono-spectral pixel component, the model can use information from all other spectral bands in the case of a multi-spectral image $(d \geq 1)$ as well. For range images $d=1$.

Let us denote another multiindex $t=(m, n, d)$ and choose a direction of movement on the image plane e.g. $t-1=(m, n-1, d), t-2=(m, n-2, d), \ldots$. The white noise component $E_{t}$ has zero mean and constant but unknown dispersion $\Omega$. We assume that the probability density of $E_{t}$ has a normal distribution independent of previous data and is the same for every time $t$. The task consists in finding the conditional prediction density $p\left(Y_{t} \mid Y^{(t-1)}\right)$ given the known process history $Y^{(t-1)}=\left\{Y_{t-1}, Y_{t-2}, \ldots, Y_{1}, Z_{t}, Z_{t-1}, \ldots, Z_{1}\right\}$ and taking its conditional mean estimation $\tilde{Y}$ for the predicted data. If a prediction error is greater than an adaptive threshold the algorithm assumes an object face edge pixel. We have chosen the conditional mean estimator for data prediction, because of its optimal properties ([3]):

$$
\tilde{Y}_{t}=E\left[Y_{t} \mid Y^{(t-1)}\right]
$$

Assuming normality of the white noise component $E_{t}$, conditional independence between pixels and an a priori probability density for the unknown model parameters chosen in the form (this normal form of a priori probability results in analytically manageable form of a posteriori probability density) 


$$
p\left(P, \Omega^{-1} \mid Y^{(0)}\right)=(2 \pi)^{-\frac{\gamma(0)}{2}}|\Omega|^{-\frac{\gamma(0)}{2}} \exp \left\{-\frac{1}{2} \operatorname{tr}\left\{\Omega^{-1}\left(\begin{array}{c}
-I \\
P
\end{array}\right)^{T} V_{0}\left(\begin{array}{c}
-I \\
P
\end{array}\right)\right\}\right\}
$$

where $V_{0}$ is a positive definite $(\beta+1) *(\beta+1)$ matrix and $\gamma(0)>d$, we have shown ([5]) that the conditional mean value is:

$$
\tilde{Y}_{t}=\hat{P}_{t-1}^{T} Z_{t}
$$

The following notation is used in (4) and (5):

$$
\begin{gathered}
\hat{P}_{t-1}=V_{z z(t-1)}^{-1} V_{z y(t-1)}, \\
V_{t-1}=\tilde{V}_{t-1}+V_{0} \\
\tilde{V}_{t-1}=\left(\begin{array}{ll}
\tilde{V}_{y y(t-1)} & \tilde{V}_{z y(t-1)}^{T} \\
\tilde{V}_{z y(t-1)} & \tilde{V}_{z z(t-1)}
\end{array}\right) . \\
\tilde{V}_{x w(t-1)}=\alpha \tilde{V}_{x w(t-2)}+X_{t-1} W_{t-1}^{T}
\end{gathered}
$$

It is easy to check (see [5]) also the validity of recursive (9). We assume slowly changing parameters, consequently these equations were modified using a constant exponential "forgetting factor" $\alpha$ to allow parameter adaptation.

$$
\dot{P}_{t}=\hat{P}_{t-1}+\left(\alpha^{2}+Z_{t}^{T} V_{z z(t-1)}^{-1} Z_{t}\right)^{-1} V_{z z(t-1)}^{-1} Z_{t}\left(Y_{t}-\hat{P}_{t-1}^{T} Z_{t}\right)^{T}
$$

If the prediction error is larger than the adaptive threshold

$$
\left|\tilde{Y}_{t}-Y_{t}\right|>\frac{2.5}{l} \sum_{i=1}^{l}\left|\tilde{Y}_{t-i}-Y_{t-i}\right|
$$

then the pixel $t$ is classified as an object edge pixel. The adaptive threshold is proportional to the local mean prediction error estimation.

\section{Locally Optimal Model Selection}

Let us assume two regression models (1) $M_{1}$ and $M_{2}$ with the same number of unknown parameters $\left(\beta_{1}=\beta_{2}=\beta\right)$ and an identical neighbour index shift sets $I_{t}$ they differ only in their forgetting factors $\alpha_{1}>\alpha_{2}$. The model $M_{1}, \alpha_{1} \approx 1$ represents homogeneous image areas while the second model better represents new information coming from crossing some face borders because it allows quicker adaptation to this new information. According to the Bayesian theory, the optimal decision rule for minimizing the average probability of decision error chooses the maximum a posteriori probability model, i.e. a model 
whose conditional probability given the past data is the highest one. Predictors used in the presented algorithm can be therefore completed as in (11):

$$
\tilde{Y}_{t}= \begin{cases}\hat{P}_{1, t-1}^{T} Z_{t} & \text { if } p\left(M_{1} \mid Y^{(t-1)}\right)>p\left(M_{2} \mid Y^{(t-1)}\right) \\ \hat{P}_{2, t-1}^{T} Z_{t} & \text { otherwise }\end{cases}
$$

where $Z_{t}$ is a data vector identical to both models. Following the Bayesian framework used in our paper and choosing uniform a priori model in the absence of contrary information, $p\left(M_{i} \mid Y^{(t-1)}\right) \sim p\left(Y^{(t-1)} \mid M_{i}\right)$, the simultaneous conditional probability density can be evaluated from

$$
p\left(Y^{(t-1)} \mid M_{i}\right)=\iint p\left(Y^{(t-1)} \mid P, \Omega^{-1}\right) p\left(P, \Omega^{-1} \mid M_{i}\right) d P d \Omega^{-1} .
$$

Under the already assumed conditional pixel independence, the analytical solution has the form

$$
p\left(M_{i} \mid Y^{(t-1)}\right)=k \Gamma\left(\frac{\gamma(t-1)-\beta+2}{2}\right)\left|V_{i, z z(t-1)}\right|^{-\frac{1}{2}} \lambda_{i, t-1}^{-\frac{\gamma(t-1)-\beta+2}{2}},
$$

where $k$ is a common constant. All statistics related to a model $M_{1}(7)-(9),(13)$ are computed using the exponential forgetting constant $\alpha_{1}$ while symmetrical statistics of the model $M_{2}$ are computed using the second constant $\alpha_{2}$. The solution of (13) uses the following notations:

$$
\begin{gathered}
\gamma(t)=\alpha_{i}^{2} \gamma(t-1)+1 \\
\lambda_{t-1}=V_{y y(t-1)}-V_{z y(t-1)}^{T} V_{z z(t-1)}^{-1} V_{z y(t-1)} .
\end{gathered}
$$

The determinant $\left|V_{z z(t)}\right|$ as well as $\lambda_{t}$ can be evaluated recursively ([5]):

$$
\begin{gathered}
\left|V_{z z(t)}\right|=\left|V_{z z(t-1)}\right| \alpha_{i}^{2 \beta}\left(1+Z_{t}^{T} V_{z z(t-1)}^{-1} Z_{t}\right) \\
\lambda_{t}=\lambda_{t-1} \alpha_{i}^{2}\left(1+\left(Y_{t}-\hat{P}_{t-1}^{T} Z_{t}\right)^{T} \lambda_{t-1}^{-1}\left(Y_{t}-\hat{P}_{t-1}^{T} Z_{t}\right)\left(\alpha_{i}^{2}+Z_{t}^{T} V_{z z(t-1)}^{-1} Z_{t}\right)^{-1}\right) .
\end{gathered}
$$

\section{Numerical Realization}

The predictors in (11) can be evaluated using updating of matrices $V_{i, t}(7)$ and their following inversion. Another possibility is the direct updating of $\hat{P}_{i, t}(9)$. To ensure the numerical stability of the solution, it is advantageous to calculate $\hat{P}_{i, t}(9)$ using a square-root filter, which guarantees the positivity of matrix (7). The filter updates directly the Cholesky square root of matrices $V_{i, t}^{-1}$.

Alternatively it is possible to use the UDU filter (a factorization into two triangular and one diagonal matrices) for this purpose. Initialisation of recursive 

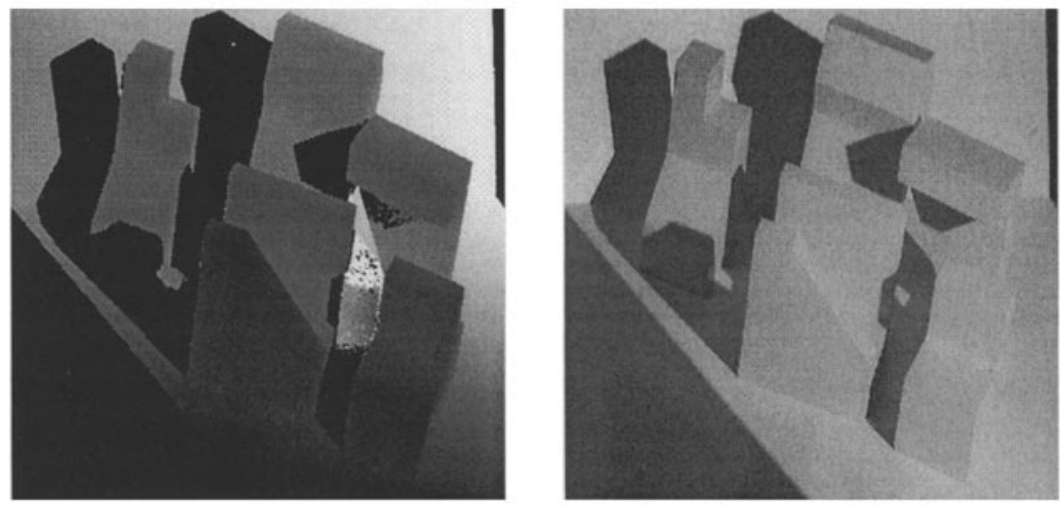

Fig. 1. ABW test image and intensity image \#0.

(9) and (17) must keep the condition of positive definiteness of matrices $V_{i, 0}$ (4). We implemented in our algorithm the uniform a priori start $V_{i, 0}=I$. This solution not only conforms with the initial lack of information at the start of algorithm, but also simplifies the calculation of the integral (12). Another possibility could be for example a local condition start, which ensures a quicker adaptation .

\section{Results}

In this section we present segmentation results of the proposed method and compare them with methods surveyed in [7]. Our goal was to properly detect single object face borders, while the performance evaluation in [7] is based on region comparison. Thus we were forced to use different performance criteria and to apply them also on test images, ground truth, and segmentation results from [7] to objectively compare the proposed segmentation algorithm performance with the state of art in the range image segmentation field. A version of our algorithm complemented with a region growing step to produce single object faces will be reported elsewhere. The performance of the methods is compared on two types real range images - the laser range finder and structured light scanner images. Objects present on these images are restricted to the objects with planar surfaces. The reason for this were difficulties of direct comparison of segmented curved surface patches. The first $512 \times 512$ test image shown on Fig. 1 was acquired from an ABW structured light scanner [11] and contains five polyhedral objects placed in a variety of angles and with different interobject spacing. Single objects have from 10 to 16 faces. The second test image (Fig. 2) with four more complex polyhedral objects (16 to 20 faces) was acquired from a Perceptron laser range finder [9]. The ABW range camera recovers depth from triangulation using structured light while the Perceptron computes the phase shift between an outgoing laser beam and its returned signal. Structured light scanner images have some shadow areas, where the sensor cannot make a 

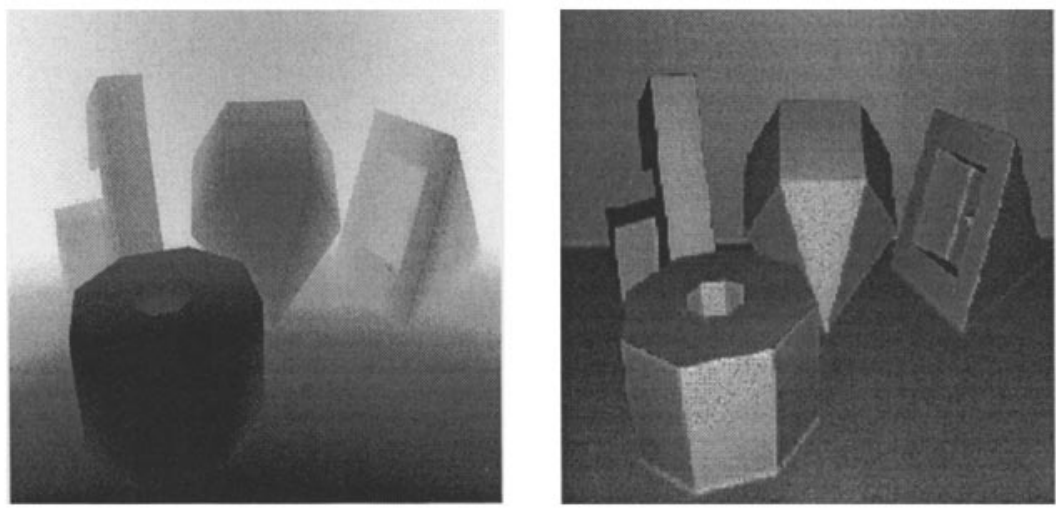

Fig. 2. Perceptron test image and intensity image \#26.

range measurement. Laser images on the other hand are more noisy than the $\mathrm{ABW}$ data and their segmentation results are reported in [7] to be worse in all performance metrics used.

Ground truth for both images were hand made by a human operator outlining the boundary of each apparent surface patch in each image and subsequently corrected by another human operator. We took over these sets for our results evaluation too. Comparison of range image segmentations to the ground truth is done using the criteria of probability of finding a correct border pixel

$$
P_{c}=\frac{\operatorname{card}\{\mathcal{T} \cap \mathcal{G}\}}{\operatorname{card}\{\mathcal{G}\}}, \quad P_{w}=\frac{\operatorname{card}\{\mathcal{T} \cap(\mathcal{I}-\mathcal{G})\}}{\operatorname{card}\{\mathcal{I}\}}
$$

and a probability of wrong border pixel detection where $\mathcal{G}, \mathcal{T}, \mathcal{I}$ are the ground truth set, the segmentation result set, and the set of all image pixels, respectively. Pixels corresponding to the contextual support set $I_{i}$ are denoted $*$ and the predicted pixel $\circ$, respectively. The segmentation results of both examples (ABW \# 0, Perceptron \# 26) are shown in Fig.3. The optimal regression models $M_{i}$ for the ABW image \# 0 were found to be: $M_{i} * \circ *$ The second tested image (Perceptron \# 26) optimal support set was found: $M_{i} *$ 。

Performance metrics used to compare segmentation algorithms in [7] were based on comparison of segmented regions. Our algorithm (A) is based on border detection between regions hence the comparison was made on region borders extracted from result images obtained using segmentation methods USF [2], WSU [6], UB [8], and UE [7]. Results in Table 1. show good performance of our method in comparison with previously published range segmenters, while being much faster than all these methods. Although we could not directly compare processing times of all evaluated methods because average processing times in [7] were measured on different platforms, we can estimate our processing time to be comparable to the quickest method UB and two orders of magnitude lower than the slowest method USF. The presented method clearly outperforms the tested methods in the overall number of wrongly detected border pixels, but in 

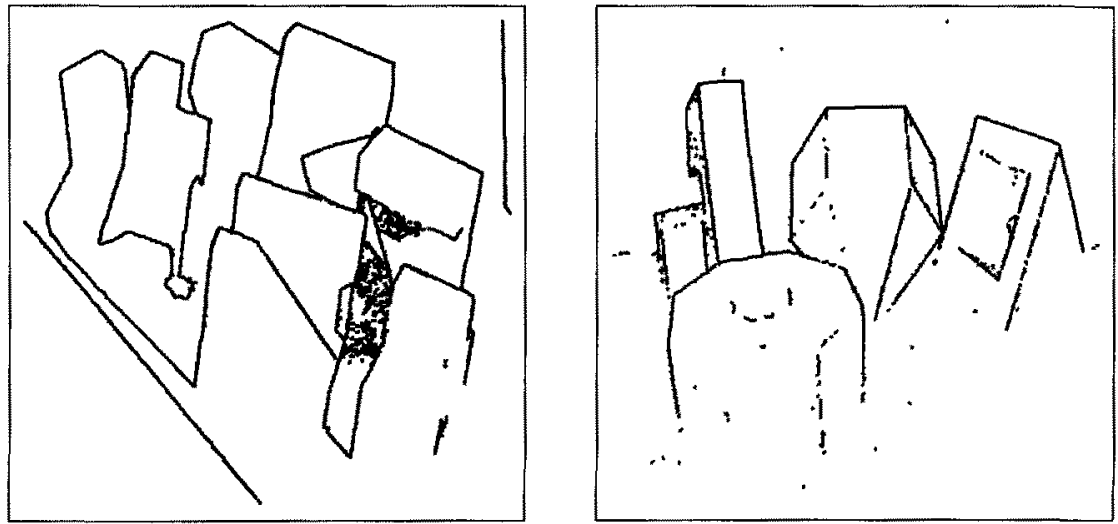

Fig. 3. ABW segmentation and Perceptron segmentation results.

Table 1. Segmentation performance criteria

\begin{tabular}{|l|c|c|c|c|}
\hline \multirow{2}{*}{ method } & \multicolumn{2}{|c|}{ ABW \# 0 } & \multicolumn{2}{|c|}{ Perceptron \# 26 } \\
\cline { 2 - 5 } & $P_{c}$ & $P_{w}$ & $P_{c}$ & $P_{w}$ \\
\hline USF & 0.69 & 0.02 & 0.69 & 0.07 \\
WSU & 0.68 & 0.009 & - & - \\
UB & 0.77 & 0.009 & 0.63 & 0.02 \\
UE & 0.86 & 0.011 & 0.62 & 0.02 \\
A & 0.64 & 0.004 & 0.52 & 0.002 \\
\hline
\end{tabular}

the same time misses some correct border pixels. Missing pixels are usually from inner object border faces. Visual comparion of results demonstrates superior quality of detected borders using our algorithm. Detected borders are clean and accurately located. Compared segmenters generally produce worse quality face borders (double edges, noisy borders, wrong face overlapping, etc.).

\section{Conclusion}

We proposed the novel efficient and robust method based on a range profile prediction modelling. A range profile is modelled using an adaptive regression model. The adaptive predictor uses spatial correlation from neighbouring data what results in improved robustness of the algorithm over rigid schemes, which are affected with outliers often present at the boundary of distinct shapes. The proposed algorithm is recursive and therefore numerically effective. A parallel implementation of the algorithm is straightforward, every image row and column can be processed independently by its dedicated processor. The numerical stability is guaranteed using the Cholesky factorization of data gathering matrices. Usual handicap of segmentation methods is their lot of application dependent 
parameters to be experimentally estimated. Some methods need nearly a dozen adjustable parameters. Our method on the other hand requires only a contextual neighbourhood selection, which can be done using Bayesian statistics of section three. The algorithm performance is demonstrated on the set of test range images used for evaluation of some other range image segmentation methods in literature [7]. Our algorithm demonstrates encouraging segmentation quality while being of an order of magnitude faster than these techniques.

The test results of the algorithm are encouraging. The proposed method was always able to find all objects present in all our experimental scenes with excellent border localization precision. Detected borders can guide a subsequent image region recognition step.

The proposed method is fully adaptive, numerically robust and still with moderate computation complexity so it can be used in an on-line virtual reality acquisition system, robot navigation system or some other image acquisition systems.

\section{References}

1. Besl, P.J., Jain, R.C.: Three-dimensional object recognition. ACM Computing Surveys 17 (1985) no.1 75-145

2. Besl, P.J., Jain, R.C.: Segmentation Through Variable-Order Surface Fitting. IEEE Trans. PAMI 10 (1988) no.2 167-192

3. Broemeling,L.D.: Bayesian Analysis of Linear Models. New York, Dekker 1985

4. Flynn, P.J. Jain,A.K.: BONSAI: 3D Object Recognition Using Constrained Search. IEEE Trans. PAMI 13 (1991) no.10 1066-1075

5. Haindl,M., Šmberová, S.: A Multispectral Image Line Reconstruction method. In: Theory \& Applications of Image Analysis. P. Johansen, S. Olsen Eds., World Scientific Publishing Co., Singapore, 1992

6. Hoffman, R.L., Jain, A.K.: Segmentation and Classification of Range Images. IEEE Trans. PAMI 9 (1987) no.5 608-620

7. Hoover, A., Jean-Baptiste, G., Jiang, X., Flynn, P.J., Bunke, H., Goldof, D.B., Bowyer, K., Eggert, D.W., Fitzgibbon, A., Fisher, R.B.: An Experimental Comparison of Range Image Segmentation Algorithms. IEEE Trans. PAMI 18 (1996) no.7 $673-689$

8. Jiang, X.Y., Bunke, H.: Fast Segmentation of Range Images into Planar Regions by Scan Line Grouping. Machine Vision and App. 7 (1994) no. 2 115-122

9. Perceptron Inc., LASAR Hardware Manual, 23855 Research Drive, Farmington Hills, Michigan, 1993

10. Sinha, S.S., Jain, R.: Handbook of Pattern Recognition and Image Processing. Wiley, New York, 1994

11. Stahs, T.G., Wahl, F.M.: Fast and Robust Data Acquisition in a Low-Cost Environment., SPIE no.1395: Close-Range Photogrammetry Meets Machine Vision (1990) 496-503, Zurich

12. Zhang, X.; Zhao, D.: Range image segmentation via edges and critical points. Proc. SPIE 2501 (1995) no. 3 1626-1637 